\title{
Special Issue on the Advances in Engineering for Women's Health
}

\author{
Raffaella De Vita ${ }^{1}$ and Jennifer Munson ${ }^{2}$ \\ ${ }^{1}$ Department of Biomedical Engineering and Mechanics, Virginia Tech, 335 Kelly Hall, 325 Stanger Street, Blacksburg, \\ VA 24061, USA ${ }^{2}$ Department of Biomedical Engineering and Mechanics, Fralin Biomedical Research Institute, Virginia Tech, \\ 349 Kelly Hall, 325 Stanger Street, Blacksburg, VA 24061, USA
}

(Published online 11 August 2021)

Women's health encompasses all physiologic and pathologic changes that occur in the female reproductive organs and supporting structures, diseases that disproportionately affect women (e.g., ovarian cancer, lipedema), and the study of diseases that affect women differently than men (e.g., heart disease). Women's health is an important yet largely understudied area of research, with many challenging and unsolved problems for biomedical engineers. Biomechanics of female reproductive organs, tissues, and cells is increasingly recognized as being crucial for women's gynecologic, reproductive, and sexual health care. New experimental, computational, and theoretical methods are being developed to prevent pregnancy-related morbidities and treat pelvic floor disorders. The field of tissue engineering is rapidly evolving to create novel fertility treatments and options, repair of degenerated or damaged tissues, and complex in vitro models that recapitulate the tumor microenvironment in femalerelated cancers (e.g., ovarian and breast cancers). The diversity in engineering applications and techniques being developed to uniquely address health issues affecting women over their lifespan is increasing at a fast pace. Building upon recent publications in the Annals of Biomedical Engineering related to women's health, ${ }^{3,4,7,9,13,16,17}$ this special issue will expand on the biomechanics of reproductive organs and their supporting structures, structural organization of pelvic organs and tissues and their functional alterations with aging and prolapse, and female-related cancers.

Female reproductive organs go through extreme changes during pregnancy and childbirth that make them unique from a biomechanical perspective. In this special issue, Grimm et al. ${ }^{11}$ presented the first review that consolidates all the published data on the forces (i.e., uterine contractions and semi-voluntary pushing)

Address correspondence to Raffaella DeVita, Department of Biomedical Engineering and Mechanics, Virginia Tech, 335 Kelly Hall, 325 Stanger Street, Blacksburg, VA 24061, USA. Electronic mail:devita@vt.edu that are involved with labor and delivery. Conway et $a l .{ }^{5}$ proposed the use of novel inflation-extension experiments over existing testing methods in order to evaluate the mechanical properties of the cervix in the murine model. The experiments were conducted by preserving the in vivo geometry and multiaxial loading of the organ as well as the interactions between the extracellular matrix and cells. In a very well-integrated experimental and computational study, Fang et al. ${ }^{10}$ provided a through characterization of the mechanical behavior of human uterus. Using spherical indentation in combination with the digital image correlation, optical coherence tomography, and inverse finite element methods, the uterus was found to be highly anisotropic and heterogeneous. Computational in silico and cellular in vitro techniques for studying the placenta and placenta-related preterm birth were discussed in an extensive review by Wheeler and Oyen ${ }^{18}$. This transient organ, which connects the mother and the developing fetus during pregnancy, can only be studied with complementary bioengineering approaches. Improved transabdominal photoacoustic imaging methods for the placenta were proposed by Huda et al. ${ }^{12}$ The new imaging methods provide increased imaging depth through optimization of light delivery.

Alterations of pelvic organs and supportive connective and muscle tissues following pregnancy and delivery, along with changes associated with age or weight gain, increase women's risk of pelvic organ prolapse. Pelvic organ prolapse occurs when any pelvic organ descends from its normal position pushing against the walls of the vagina, causing concomitant pelvic floor disorders (e.g., urinary incontinence). In this special issue, Dahal et al. ${ }^{6}$ presented the first quantitative analysis of elastin fibers for the vaginal wall. The authors conducted their study using mice that have aberrant elastin metabolism leading to pelvic organ prolapse. Donaldson et al. ${ }^{8}$ offered an exhaustive review of the mechanical properties of the uter- 
osacral ligaments, the connective tissues that support the uterus and apical vagina within the female pelvis. These anatomical structures, together with the pelvic floor muscles, are implicated with the development and treatment of pelvic floor disorders. In the study by Rieger et al., ${ }^{19}$ age-related degeneration was shown to disproportionally impact pelvic floor muscles relative to the appendicular muscles using cadaveric specimens. This conclusion was based on tissue and cell level alterations in the intrinsic components of the pelvic floor muscles (i.e., contractile myofibers, non-contractile extracellular matrix, and resident stem cells). Fascicle arrangements of two crucial muscles that serve to stabilize the abdominal and pelvic organs, the coccygeus and levatorani, were quantified by Routzong et al. ${ }^{20}$ using close-range photogrammetry from human cadavers, providing novel data for accurate simulations of the female pelvic floor. Three-dimensional finite element simulations of the human female urethra constructed from sequential magnetic resonance images of a healthy nulliparous subject were conducted by Attari et al. ${ }^{2}$ The computational model was employed to investigate individual contributions of urethral striated and smooth muscles and vascular plexus on the urethral closure pressure for maintaining continence or initiating micturition.

Cancers that affect women's organs are some of the most common cancers in the United States. Though breast cancer is the most well-known, gynecologic cancers (e.g., cervical, ovarian, uterine) are far more lethal yet comparatively under-funded and -researched. ${ }^{15}$ As with breast cancer, biomedical engineers are creating solutions for the study and therapeutic screening of these women's cancers. Amirghasemi et al. ${ }^{1}$ presented a comprehensive review of three- dimensional micro-engineering platforms for anti-cancer drug discovery, with an emphasis on current techniques being used to improve the survival rate of women. These therapeutic tissue engineered testing platforms can offer flexibility of both drug testing and patient-specific evaluation which can lead to better patient care overall. In a global health-oriented women's health publication, Schwartz et al. highlight genetic differences in a population of Ethiopian women with breast cancers as compared to European women and African-American women. ${ }^{14}$ Interestingly of the set of differentially expressed genes, mutations in extracellular matrix encoding genes implicated in metastasis were identified in the Ethiopian cohort. Characterization studies, like this, are important for development of tissue engineered models and therapeutic identification that is inclusive of ethnicity and race, in addition to sex. Better representation of intersectional groups is an important step forward as we develop engineering approaches for women's health, in that they further address health disparities in underrepresented groups.

We are grateful to Prof. Stefan Duma, Editor-inChief of the Annals of Biomedical Engineering, for accepting our proposal for this special issue and his full support throughout the editorial procedure. We thank Prof. Joel Stitzel, Deputy Editors-in-Chief of the Annals of Biomedical Engineering for his guidance on the peer review process. We are also grateful to all the authors who contributed to this issue and to all the reviewers for providing invaluable feedback on the manuscripts. It is our hope that this special issue will serve to further advance the field of engineering for women's health, a newly emerging field that offers many exciting opportunities for scientific research.

\section{REFERENCES}

${ }^{1}$ Amirghasemi, F., E. Adjei-Sowah, B. A. Pockaj, and M. Nikkhah. Microengineered 3D tumor models for anticancer drug discovery in female-related cancers. Ann. Biomed. Eng. 2021. https://doi.org/10.1007/s10439-020-02 704-9.

${ }^{2}$ Attari, A., J. O. DeLancey, and J. A. Ashton-Miller. On structure-function relationships in the female human urethra: a finite element model approach. Ann. Biomed. Eng. 2021. https://doi.org/10.1007/s10439-021-02765-4.

${ }^{3}$ Baah-Dwomoh, A., M. Alperin, M. Cook, and R. De Vita. Mechanical analysis of the uterosacral ligament: swine vs. human. Ann. Biomed. Eng. 46(12):2036-2047, 2018.

${ }^{4}$ Claure, I., D. Anderson, C. M. Klapperich, W. Kuohung, and J. Y. Wong. Biomaterials and contraception: promises and pitfalls. Ann. Biomed. Eng. 48(7):2113-2131, 2020.

${ }^{5}$ Conway, C. K., A. Varghese, M. Mahendroo, and K. S. Miller. The role of biaxial loading on smooth muscle contractility in the nulliparous murine cervix. Ann. Biomed. Eng. 2021. https://doi.org/10.1007/s10439-021-02778-z.

${ }^{6}$ Dahal, S., M. Kuang, A. Rietsch, R. S. Butler, A. Ramamurthi, and M. S. Damaser. Quantitative morphometry of elastic fibers in pelvic organ prolapse. Ann. Biomed. Eng. 2021. https://doi.org/10.1007/s10439-021-02760-9.

${ }^{7}$ Dhandapani, R., A. Subramanian, and S. Sethuraman. ECM-Mimetic multiresponsive nanobullets targeted against metastasizing circulating tumor clusters in breast cancer. Ann. Biomed. Eng. 48(2):568-581, 2020.

${ }^{8}$ Donaldson, K., A. Huntington, and R. De Vita. Mechanics of uterosacral ligaments: current knowledge, existing gaps, and future directions. Ann. Biomed. Eng. 2021. https://doi.org/10.1007/s10439-021-02755-6.

${ }^{9}$ Donnely, E., M. Griffin, and P. E. Butler. Breast reconstruction with a tissue engineering and regenerative medicine approach (systematic review). Ann. Biomed. Eng. 48(1):9-25, 2020.

${ }^{10}$ Fang, S., J. McLean, L. Shi, J. Y. Vink, C. P. Hendon, and K. M. Myers. Anisotropic mechanical properties of the human uterus measured by spherical indentation. Ann. Biomed. Eng. 2021. https://doi.org/10.1007/s10439-021-02 769-0. 
${ }^{11}$ Grimm, M. J. Forces involved with labor and delivery-a biomechanical perspective. Ann. Biomed. Eng. 2021. http s://doi.org/10.1007/s10439-020-02718-3.

${ }^{12}$ Huda, K., K. F. Swan, C. T. Gambala, G. C. Pridjian, and C. L. Bayer. Towards transabdominal functional photoacoustic imaging of the placenta: improvement in imaging depth through optimization of light delivery. Ann. Biomed. Eng. 2021. https://doi.org/10.1007/s10439-021-02777-0.

${ }^{13}$ Huntington, A., E. Rizzuto, S. Abramowitch, Z. Del Prete, and R. De Vita. Anisotropy of the passive and active rat vagina under biaxial loading. Ann. Biomed. Eng. 47(1):272281, 2019.

${ }^{14}$ Schwartz, A. D., A. Adusei, S. Tsegaye, C. A. Moskaluk, S. S. Schneider, M. O. Platt, D. Seifu, S. Peyton, and C. C. Babbitt. Genetic mutations associated with hormone-positive breast cancer in a small cohort of Ethiopian women. Ann. Biomed. Eng. 2021. https://doi.org/10.1007/s10439-0 21-02800-4.

${ }^{15}$ Spencer, R. J., L. W. Rice, C. Ye, K. Woo, and S. Uppal. Disparities in the allocation of research funding to gynecologic cancers by funding to lethality scores. Gynecol Oncol. 152(1):106-111, 2019.

${ }^{16}$ Metsiou D. N., K. E. Siatis, E. Giannopoulou, D. J. Papachristou, H. P. Kalofonos, A. Koutras and G. Athanassiou. The Impact of Anti-tumor Agents on ERPositive MCF-7 and HER2-Positive SKBR-3 Breast Can- cer Cells Biomechanics. Ann. Biomed. Eng. 47(1):17111724, 2019.

${ }^{17}$ Sittadjody S., K. M. Enck, A. Wells, J. J. Yoo, A. Atala, J. M. Saul and E. C. Opara. Encapsulation of mesenchymal stem cells in 3D ovarian cell constructs promotes stable and long-term hormone secretion with improved physiological outcomes in a syngeneic rat model. Ann. Biomed. Eng. 48:1058-1070, 2020.

${ }^{18}$ Wheeler, M. L., Oyen, M. L. Bioengineering approaches for placental research. Ann. Biomed. Eng. 2021. https://d oi.org/10.1007/s10439-020-02714-7.

${ }^{19}$ Rieger, M., Duran, P., Cook, M., Schenk, S., Shah, M., Jacobs, M., Christman, K., Kado, D.M., and M. Alperin. Quantifying the effects of aging on morphological and cellular properties of human female pelvic floor muscles. Ann. Biomed. Eng. 2021. https://doi.org/10.1007/s10439-0 21-02748-5.

${ }^{20}$ Routzong, M. R., Cook, M. S., Barone, W., Abramowitch, S.D., and M. Alperin. Novel application of photogrammetry to quantify fascicle orientations of female cadaveric pelvic floor muscles. Ann. Biomed. Eng. 2021. https://doi. org/10.1007/s10439-021-02747-6.

Publisher's Note Springer Nature remains neutral with regard to jurisdictional claims in published maps and institutional affiliations. 